\title{
Pupil Dilatation with Tropicamide. The Effects on Acuity, Accommodation and Refraction
}

\author{
D. M. I. MONTGOMERY ${ }^{1}$ and C. J. MACEWAN ${ }^{2}$ \\ Glasgow and Dundee.
}

\begin{abstract}
Summary
The effect of pupil dilatation with tropicamide $1 \%$ on visual acuity and accommodation was assessed in 100 eyes of 52 consecutive patients attending the general ophthalmic outpatient clinic.

Snellen visual acuity remained unchanged in 55 eyes and deteriorated by one line in 41 eyes. The remaining four eyes deteriorated by two lines. Tropicamide gave rise to a reduction in the amplitude of accommodation which tended to vary inversely with the age of the patient. However, all patients who wore reading glasses for presbyopia were still able to read when their pupils had been dilated.
\end{abstract}

Many drugs are used routinely in ophthalmic practice to dilate the pupil for adequate examination of the ocular fundus. Selection may be based on the speed of onset and duration of action of the drug as well as on the presence and severity of any unwanted effects such as cycloplegia. Tropicamide is a drug often favoured because of its short duration of action and relatively greater mydriatic than cycloplegic effect.

Concern is often expressed with regard to adverse effects of pupil dilatation on vision which might affect patients' ability to travel home safely or to return to work. Tropicamide has recently been shown to have little effect on the distance visual acuity of a group of healthy young volunteers. ${ }^{1}$ However, no attempt has hitherto been made to quantify visual impairment associated with pupil dilatation in the, often elderly, clinic population. The aim of this study was to determine the effect of tropicamide dilatation on visual acuity and accommodation in such a population.

\section{Materials and methods}

Tropicamide $1 \%$ was used to dilate the pupils of 100 eyes of 52 consecutive patients who required fundoscopy during attendance at the general ophthalmology outpatient clinic. The mean patient age was 38 years (range 6-84 years). Prior to dilatation retinoscopy was carried out and the uncorrected and best corrected visual acuities were recorded using a standard six metre Snellen chart. The amplitude of accommodation was measured for each eye using the RAF rule. One drop of tropicamide $1 \%$ was instilled into each eye producing sufficient dilatation in all cases for satisfactory examination after thirty minutes. At this time, the uncorrected and corrected acuities were again recorded. Retinoscopy was repeated and any change in refraction was

From: ${ }^{1}$ Registrar in Ophthalmology, Tennent Institute of Ophthalmology, Western Infirmary, Glasgow. ${ }^{2}$ Senior Registrar in Ophthalmology, Eye Department. Ninewells Hospital, Dundee.

Correspondence to: Dr DMI Montgomery, Tennent Institute of Ophthalmology, Western Infirmary, Glasgow G11 6NT. 
noted. The amplitude of accommodation was again measured.

Eyes were grouped according to their refractive state namely emmetropes (33 eyes), myopes (27 eyes) and hypermetropes (40 eyes). For the purpose of this study hypermetropes and myopes were defined as those eyes in which retinoscopy revealed refractive errors of greater than 0.5 dioptre spherical equivalent. The remainder were classed as emmetropic although some eyes with low degrees of mixed astigmatism were included in this group.

\section{Results}

\section{Visual acuity}

Thirty minutes after the instillation of tropicamide $1 \%$, corrected visual acuity was unchanged in 55 eyes; 41 eyes demonstrated a drop of one line and four eyes a drop of two

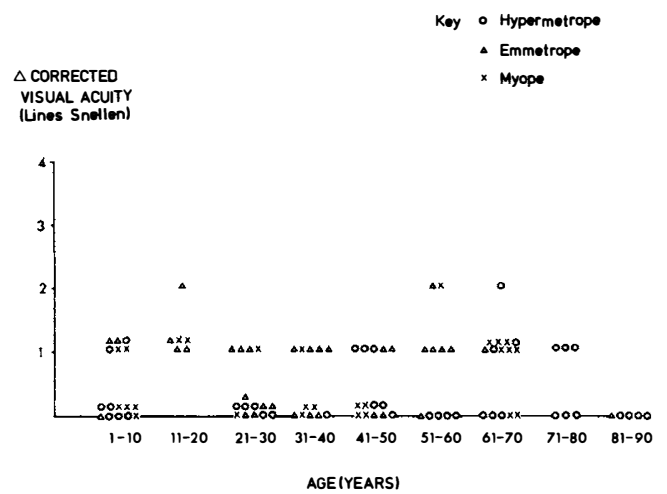

Fig. 1

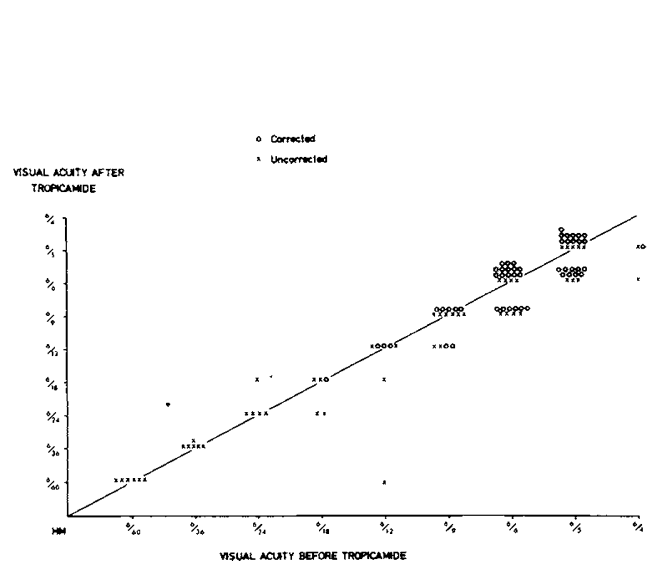

Fig. 3 lines of Snellen acuity (Fig.1). There was no significant difference between the results obtained for hypermetropes, myopes or emmetropes $\left(\chi^{2}\right.$ test; $\left.0.1<\mathrm{p}<0.2\right)$. The uncorrected visual acuity remained unchanged in 73 eyes; fell by one line in 20 eyes; fell by two lines in four eyes and by three lines in two eyes. The acuity of one eye deteriorated by four lines. Emmetropic eyes showed a significantly greater change than myopes or hypermetropes $\left(\chi^{2}\right.$ test $\left.0.02<\mathrm{p}<0.05\right)$ (Figs. 2 and $3)$. The statistical method described by Rosner $^{2}$ was applied to take account of any possible correlation between the two eyes of a given subject.

The degree and axis of any astigmatism present did not appear to be related to the changes in visual acuity.

\section{Accommodation}

The mean amplitude of accommodation

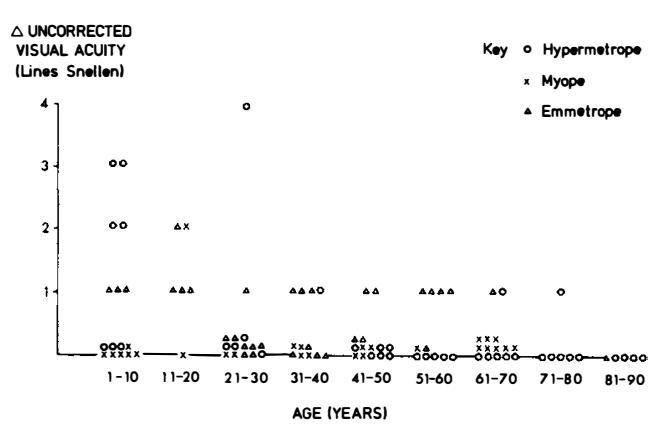

Fig. 2

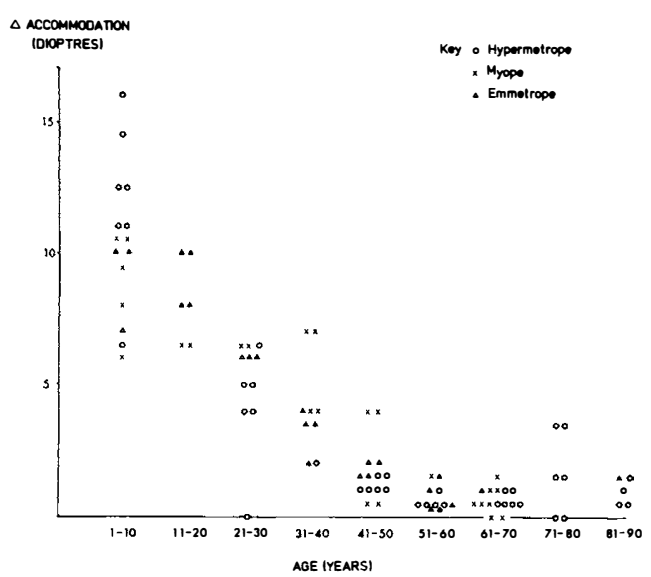

Fig. 4 


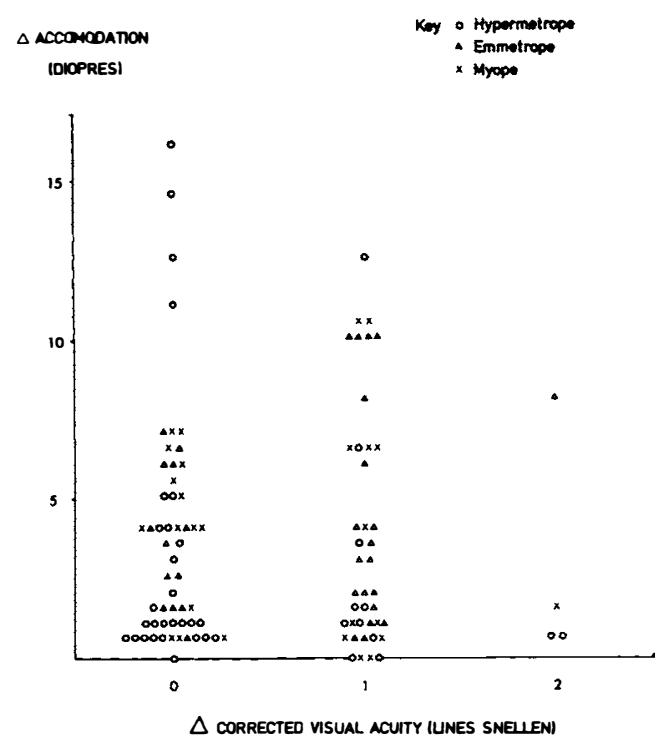

Fig. 5

before the administration of tropicamide was six dioptres (range 1-20) and after tropicamide was three dioptres (range $0.5-7$ ). The change in accommodation tended to vary inversely with age (Fig.4). Twenty nine patients wore reading glasses for presbyopia and none found difficulty with reading following the instillation of tropicamide. Only 14 patients were rendered unable to read at a comfortable distance. The change in accommodation was unrelated to the change in distance acuity (Fig.5).

\section{Refraction}

The retinoscopy readings remained unaltered in 83 eyes, increased by one dioptre in ten eyes and by 1 to 3 dioptres in the remaining seven eyes. All patients in the last group were under the age of 25 years. Only eight of the 16 children under the age of ten years demonstrated any change in retinoscopy.

\section{Discussion}

Tropicamide, a parasympatholytic agent, is widely used for pupil dilatation because of its rapid onset and short duration of action. This effect takes approximately 30 minutes to become fully effective, stays at its peak for another 10-20 minutes and lasts up to four hours. ${ }^{3}$ Its cycloplegic action has largely worn off after one hour. ${ }^{4,5}$ Although it has been suggested that the $1 \%$ solution offers no advantage over the $0.5 \%$ solution for dilatation $^{5}$ we used the $1 \%$ solution to demonstrate the maximum effect of the drug after only one drop. The examination began at 30 minutes to coincide with the time of maximum drug action.

Theoretically, the instillation of tropicamide might be expected to impair vision by two main mechanisms. Firstly, mydriasis might make manifest a refractive error previously masked by the stenopaeic effect of a small pupil. The depth of field will be reduced while spherical aberration and glare may be introduced. Secondly, the documented cycloplegic effect of tropicamide,,$^{3,4,5,7,8}$ by reducing accommodation, could impair near vision in all patients except uncorrected myopes who do not require accommodation for near vision. Moreover latent hypermetropia may be made manifest resulting in impaired distance acuity.

In practice, we found that tropicamide $1 \%$, while producing pupillary dilatation adequate for fundoscopy, caused little visual disturbance as assessed by the methods described. This may reflect the rather variable and incomplete cycloplegia produced by a single drop of this drug.

Corrected visual acuity in particular was unaffected in the great majority of patients. Those who did suffer an impairment in acuity would not have been predicted on the basis of either age or the refractive state of the eyes.

Uncorrected acuity also showed little difference between the pre- and post-dilatation levels. This assessment was included as patients may arrive at a clinic without glasses and suffer a reduction in vision due to a previously masked refractive error. One patient in this study did suffer a significant reduction in uncorrected acuity in one eye, falling from $6 / 12$ to $6 / 60$. This was a young hypermetropic man who had with the rule astigmatism (the fellow eye being amblyopic) and he illustrates that some patients may be diminishing the effect of their refractive error by means of accommodation or a small pupil.

The uncorrected acuity of emmetropes was more affected than that of hypermetropes or myopes. This can be explained on the basis 
that the emmetropes initially had better acuities. Sixty-three per cent of eyes with a refractive error had an initial acuity of $6 / 18$ or worse compared with $6 / 5$ in some of the emmetropes and were therefore less sensitive to smaller increments on the Snellen chart (eg 6/36 to $6 / 60$ compared with $6 / 5$ to $6 / 6$ ).

The change in accommodation was noted to vary inversely with the age of the patients, reflecting the reduced residual accommodation associated with ageing. It should be remembered, however, that some apparent loss of amplitude of accommodation may be due to loss of depth of field asssociated with a large pupillary aperture. This may explain the discrepancy between the retinoscopic findings and the accommodation measurements as well as the lack of correlation between the changes in distance acuity and accommodation.

In conclusion, it should be pointed out that while the clinical tests of visual function employed in this study are useful indicators of the level of potential subjective difficulties for the patient they should not be regarded as foolproof. For example, a patient with early lens opacities may achieve $6 / 9$ acuity with dilated pupils in the subdued lighting of the clinic but be quite incapacitated by glare on entering bright sunlight. It is unlikely that more sophisticated visual assessment, such as contrast sensitivity testing, would be any more reliable in predicting such disability. For this reason it is recommended that while the doctor may readily advise his patient of the likelihood of visual disturbance following pupil dilatation, in any individual case the onus of responsibility for his own safety, and the safety of others, must remain with the patient whose subjective assessment of any form of visual impairment is likely to be ultimately the most sensitive indicator of risk to driving and other abilities.

We wish to thank Professor W. S. Foulds for his encouragement and helpful criticism of this work and Dr D. Allen for his assistance with the statistical analysis.

\section{References}

${ }^{1}$ Nelson ME and Orton HP: Counteracting the Effects of Mydriatics. Does it benefit the patient? Arch Ophthalmol 1987, 105: 486-9.

2 Rosner B: Statistical methods in ophthalmology: an adjustment for the interclass correlation between eyes. Biometrics 1982, 38: 105-14.

${ }^{3}$ Hiatt RL and Jerkins G: Comparisons of Atropine and Tropicamide in Esotropia. Ann Ophthalmol 1983, 4: 341-3.

${ }^{4}$ Milder B: Tropicamide as a Cycloplegic Agent. Arch Ophthalmol 1961, 4: 341-3.

${ }^{5}$ Gettes BC and Belmont O: Tropicamide: Comparative Cycloplegic Effects. Arch Ophthalmol 1961, 66: 70-4.

${ }^{6}$ Davidson SI: Mydriatic and Cycloplegic Drugs Trans Ophthalmol Soc UK 1976, 96: 327-9.

${ }^{7}$ Gettes BC: Three New Cycloplegic Drugs. Arch Ophthalmol 1954, 51: 467-72.

${ }^{8}$ Gettes BC: Tropicamide: A New Cycloplegic Mydriatic. Arch Ophthalmol 1961, 65: 48-51. 